\title{
SYMMETRIC AND SELFADJOINT RELATIONS IN KREĬN SPACES II
}

\author{
A. DIJKSMA and H. S. V. DE SNOO
}

\section{Introduction}

In [11] Langer has obtained a spectral function for a class of selfadjoint operators in Krein spaces, namely the definitizable operators. Selfadjoint operators in Pontryagin spaces are definitizable, and hence always possess a spectral function. His arguments were made available in [12]. Other proofs were given in for instance [3], [4], [8] and [9].

In the present paper we shall show that Langer's constructions can be adapted, in order to obtain similar results for selfadjoint relations in Kreĭn spaces which are definitizable. This paper is a continuation of [6], where we gave some basic facts concerning relations in inner product spaces.

Some preliminary results are to be found in Section 2. We develop an operational calculus for subspaces (closed linear relations) in Banach spaces in Section 3, via contour integration of the resolvent operator. Definitizable subspaces in Krel̆n spaces are introduced in Section 4. Analytic properties of the resolvent operator of a selfadjoint definitizable subspace are derived in Section 5. For definitions and notations we refer to [6].

\section{Preliminary results}

In this section we will collect a number of results, that will be used later on. Let $\mathbf{K}$ be a linear space over $\mathbf{C}$ and let $A$ be a linear relation in $\mathbf{K}^{2}$. For any $2 \times 2$ matrix

$$
M=\left(\begin{array}{ll}
\alpha & \beta \\
\gamma & \delta
\end{array}\right)
$$

with complex entries $\alpha, \beta, \gamma, \delta$ we define the relation $M A$ by

$$
M A=\{\{\alpha f+\beta g, \gamma f+\delta g\} \mid\{f, g\} \in A\} .
$$

The mapping $A \rightarrow M A$ is sometimes called a transformer, see [13]. In terms of trans- 
formers we have

and

$$
\begin{aligned}
A-\lambda & =\left(\begin{array}{rr}
1 & 0 \\
-\lambda & 1
\end{array}\right) A, \quad \lambda \in \mathbf{C}, \\
\alpha A & =\left(\begin{array}{ll}
1 & 0 \\
0 & \alpha
\end{array}\right) A, \quad \alpha \in \mathbf{C},
\end{aligned}
$$

$$
A^{-1}=\left(\begin{array}{ll}
0 & 1 \\
1 & 0
\end{array}\right) A
$$

If for $\mu, \zeta \in \mathbf{C}$ we define

and

$$
C_{\mu, \zeta}(A)=\{\{g-\mu f, g-\zeta f\} \mid\{f, g\} \in A\}
$$

$$
F_{\mu, \zeta}(A)=\{\{g-f, \mu g-\zeta f\} \mid\{f, g\} \in A\}
$$

then $C_{\mu, \zeta}$ and $F_{\mu, \zeta}$ are transformers, corresponding to

$$
\left(\begin{array}{ll}
-\mu & 1 \\
-\zeta & 1
\end{array}\right) \text { and }\left(\begin{array}{ll}
-1 & 1 \\
-\zeta & \mu
\end{array}\right)
$$

respectively. Note that for $\zeta=\bar{\mu}$ these give the Cayley transform $C_{\mu}$ and inverse Cayley transform $F_{\mu}$, respectively. The following property is obvious, but has a number of interesting consequences.

Transformer property. If $A \subset \mathbf{K}^{2}$ is a linear relation, and $M$ and $N$ are $2 \times 2$ matrices with complex entries, then

For example we have

$$
M(N A)=(M N) A .
$$

$$
-\frac{v-\mu}{\mu-\zeta}\left(C_{\mu, \zeta}(A)-\frac{v-\zeta}{v-\mu}\right)=C_{\mu, v}(A), \quad v \neq \mu, \zeta \neq \mu .
$$

Note that this equality holds for all $\zeta, \zeta \neq \mu$. In particular we have

$$
-\frac{v-\mu}{\mu-\bar{\mu}}\left(C_{\mu}(A)-\frac{v-\bar{\mu}}{v-\mu}\right)=C_{\mu, v}(A), \quad \mu \in \mathbf{C} \backslash \mathbf{R}, v \neq \mu .
$$

Now using the identity

we obtain

$$
C_{\mu, \zeta}(A)=\left(C_{\zeta, \mu}(A)\right)^{-1}
$$

$$
C_{\mu}(A)=\frac{v-\bar{\mu}}{v-\mu}-\frac{(\mu-\bar{\mu})(v-\bar{v})}{(\mu-v)^{2}}\left(C_{v}(A)-\frac{\mu-\bar{v}}{\mu-v}\right)^{-1}, \quad v \neq \mu .
$$

Another simple consequence of the transformer property is

and in particular

$$
C_{\mu, \zeta}(A)=I+(\mu-\zeta)(A-\mu)^{-1},
$$

$$
C_{\mu}(A)=I+(\mu-\bar{\mu})(A-\mu)^{-1} \text {. }
$$


This leads to

$$
\left(C_{\mu}(A)-\frac{\lambda-\bar{\mu}}{\lambda-\mu}\right)^{-1}=\frac{\mu-\lambda}{\mu-\bar{\mu}}\left(I+(\lambda-\mu)(A-\lambda)^{-1}\right), \quad \mu \in \mathbf{C} \backslash \mathbf{R}, \lambda \neq \mu,
$$

or equivalently

$$
\left(A-\frac{\zeta \mu-\bar{\mu}}{\zeta-1}\right)^{-1}=\frac{1-\zeta}{\mu-\bar{\mu}}\left(I+(\zeta-1)\left(C_{\mu}(A)-\zeta\right)^{-1}\right), \quad \mu \in \mathbf{C} \backslash \mathbf{R}, \zeta \neq 1 .
$$

Finally the transformer property shows

$$
(A-\lambda)^{-1}=-\frac{1}{\lambda-\alpha} I-\frac{1}{(\lambda-\alpha)^{2}}\left((A-\alpha)^{-1}-\frac{1}{\lambda-\alpha}\right)^{-1}, \quad \lambda \neq \alpha .
$$

Remark. If $\{h, k\} \in A$, then $\{h, \beta k+\alpha h\} \in \beta A+\alpha, \quad\{h, \delta k+\gamma h\} \in \delta A+\gamma$, so that $\{\alpha h+\beta k, \gamma h+\delta k\} \in(\delta A+\gamma)(\beta A+\alpha)^{-1}$. This shows

$$
\left(\begin{array}{ll}
\alpha & \beta \\
\gamma & \delta
\end{array}\right) A \subset(\delta A+\gamma)(\beta A+\alpha)^{-1}
$$

In general this inclusion will be strict. To see this consider $A=\{0\} \times \mathbf{K}$, then

while

$$
\left(\begin{array}{ll}
\alpha & \beta \\
\gamma & \delta
\end{array}\right) A=\{\{\beta h, \delta h\} \mid h \in \mathbf{K}\}
$$

$$
\begin{aligned}
(\delta A+\gamma)(\beta A+\alpha)^{-1} & =\mathbf{K}^{2}, \quad \beta \neq 0, \\
& =A, \quad \beta=0 .
\end{aligned}
$$

Proposition 2.1. Let $M=\left(\begin{array}{ll}\alpha & \beta \\ \gamma & \delta\end{array}\right)$ be invertible and let $A \subset \mathbf{K}^{2}$ be a linear relation.

(i) If $\delta \neq 0$, then $v\left((M A)^{r}\right)=v\left(\left(\left(\begin{array}{ll}\delta & 0 \\ \gamma & \delta\end{array}\right) A\right)^{r}\right)$.

(ii) If $\gamma \neq 0$, then $v\left((M A)^{r}\right)=v\left(\left(\left(\begin{array}{ll}0 & \gamma \\ \gamma & \delta\end{array}\right) A\right)^{r}\right)$.

Proof. We consider case (i). Let $h_{0} \in v\left((M A)^{r}\right)$, then there exist elements $h_{1}, \ldots, h_{r-1}, h_{r}=0$ in $\mathbf{K}$ with $\left\{h_{i}, h_{i+1}\right\} \in M A, i=0, \ldots, r-1$. Hence

$$
\left\{\delta h_{i}-\beta h_{i+1},-\gamma h_{i}+\alpha h_{i+1}\right\} \in A,
$$

which implies that

$$
\left\{\delta h_{i}-\beta h_{i+1}, \frac{\operatorname{det} M}{\delta} h_{i+1}\right\} \in\left(A+\frac{\gamma}{\delta}\right)=\left(\begin{array}{ll}
\delta & 0 \\
\gamma & \delta
\end{array}\right) A, \quad i=0, \ldots, r-1 .
$$

For $j=1$ it is clear that

$$
\left\{h_{r-j}, 0\right\} \in\left(A+\frac{\gamma}{\delta}\right)^{j}
$$


Assume this is true for $j=1, \ldots, p-1$. Then putting $i=r-p$ in (2.1) we obtain

$$
\left\{\delta h_{r-p}-\beta h_{r-p+1}, \frac{\operatorname{det} M}{\delta} h_{r-p+1}\right\} \in A+\frac{\gamma}{\delta} \text {. }
$$

We combine this with (2.2) for $j=p-1$ and find that

$$
\left\{\delta h_{r-p}-\beta h_{r-p+1}, 0\right\} \in\left(A+\frac{\gamma}{\delta}\right)^{p} .
$$

Again using (2.2) with $j=p-1$ we have

$$
\left\{h_{r-p}, 0\right\} \in\left(A+\frac{\gamma}{\delta}\right)^{p}
$$

which proves (2.2) for $j=p$. We conclude $h_{0} \in v\left(\left(\left(\begin{array}{ll}\delta & 0 \\ \gamma & \delta\end{array}\right) A\right)^{r}\right)$, and thus

$$
v\left((M A)^{r}\right) \subset v\left(\left(\left(\begin{array}{ll}
\delta & 0 \\
\gamma & \delta
\end{array}\right) A\right)^{r}\right) \text {. }
$$

As this inclusion holds for all linear relations $A \subset \mathbf{K}^{2}$, we also have

where

$$
v\left(A^{r}\right) \subset v\left((N A)^{r}\right),
$$

and consequently

$$
N=\left(\begin{array}{cc}
\frac{S^{2}}{\operatorname{det} M} & -\frac{\beta \delta}{\operatorname{det} M} \\
0 & 1
\end{array}\right)=\left(\begin{array}{ll}
\delta & 0 \\
\gamma & \delta
\end{array}\right) M^{-1},
$$

$$
v\left(A^{r}\right) \subset v\left((N A)^{r}\right) \subset v\left(A^{r}\right),
$$

which shows $v\left(A^{r}\right)=v\left((N A)^{r}\right)$, but then

$$
v\left((M A)^{r}\right)=v\left(\left(\left(\begin{array}{ll}
\delta & 0 \\
\gamma & \delta
\end{array}\right) A\right)^{r}\right)
$$

Case (ii) is proved analogously.

Corollary. Let $A \subset \mathbf{K}^{2}$ be a linear relation and $\mu \in \mathbf{C} \backslash \mathbf{R}$. Then

and

$$
v\left((A-\lambda)^{r}\right)=v\left(\left(C_{\mu}(A)-\frac{\lambda-\bar{\mu}}{\lambda-\mu}\right)^{r}\right), \quad \lambda \neq \mu,
$$

$$
A^{r}(0)=v\left(\left(C_{\mu}(A)-I\right)^{r}\right) .
$$

Note that corresponding Jordan chains have the same length. For later reference we observe

and

$$
\lambda \in \sigma_{p}(A) \Leftrightarrow \frac{\lambda-\bar{\mu}}{\lambda-\mu} \in \sigma_{p}\left(C_{\mu}(A)\right), \quad \lambda \neq \mu,
$$

$$
\infty \in \tilde{\sigma}_{p}(A) \Leftrightarrow 1 \in \sigma_{p}\left(C_{\mu}(A)\right) .
$$


Here $\tilde{\sigma}_{p}(A)$ denotes the extended point-spectrum of $A$, i.e., $\tilde{\sigma}_{p}(A)=\sigma_{p}(A) \cup\{\infty\}$ if $A(0) \neq\{0\}$ and $\tilde{\sigma}_{p}(A)=\sigma_{p}(A)$ if $A(0)=\{0\}$.

From now on we will assume that $\mathbf{K}$ is a Banach space. Recall that if $A \subset \mathbf{K}^{2}$ is a subspace, i.e., a closed linear relation, then its resolvent set is defined by

$$
\varrho(A)=\left\{\lambda \in \mathbf{C} \mid(A-\lambda)^{-1} \in[\mathbf{K}]\right\} .
$$

Note that for any subspace $A \subset \mathbf{K}^{2}$ with non-empty resolvent set we have

$$
\begin{gathered}
R_{A}(\lambda)(A-\lambda)=I_{\mathbf{D}(A)}, \quad \lambda \in \varrho(A), \\
(A-\lambda) R_{A}(\lambda)=I+\{\{0, \varphi\} \mid \varphi \in A(0)\}, \quad \lambda \in \varrho(A) .
\end{gathered}
$$

Proposition 2.2. Let $A \subset \mathbf{K}^{2}$ be a subspace with $\varrho(A) \neq \emptyset$. For any $r \in \mathbf{N}$ we have

(i) $A^{r}=\left\{\left\{R_{A}(\lambda)^{r} \chi,\left(I+\lambda R_{A}(\lambda)\right)^{r} \chi\right\} \mid \chi \in \mathbf{K}\right\}, \quad \lambda \in \varrho(A)$,

(ii) $\quad R_{A}(\lambda)^{r} A^{r} \subset\left(I+\lambda R_{A}(\lambda)\right)^{r} \subset A^{r} R_{A}(\lambda)^{r}, \quad \lambda \in \varrho(A)$,

(iii) $R_{A}(\lambda)^{r} A^{r}=\left.\left(I+\lambda R_{A}(\lambda)\right)^{r}\right|_{\mathbf{D}\left(A^{r}\right),}, \quad \lambda \in \varrho(A)$,

(iv) $R_{A}(\lambda)^{r} A^{r} R_{A}(\mu)^{r}=\left(I+\lambda R_{A}(\lambda)\right)^{r} R_{A}(\mu)^{r}, \quad \lambda, \mu \in \varrho(A)$,

$$
=\left(\frac{\lambda R_{A}(\lambda)-\mu R_{A}(\mu)}{\lambda-\mu}\right)^{r}, \quad \lambda \neq \mu .
$$

Proof. Note that it follows from (ii) that $R_{A}(\lambda)^{r} A^{r}$ is a bounded linear operator, and its domain is $\mathbf{D}\left(A^{r}\right)$. Hence (iii) is an immediate consequence of the first inclusion of (ii). It follows from (i) that $R_{A}(\lambda)^{r}$ maps $\mathbf{K}$ onto $\mathbf{D}\left(A^{r}\right)$. Hence the first equality in (iv) follows from (iii). The second equality in (iv) follows from

$$
\left(I+\lambda R_{A}(\lambda)\right) R_{A}(\mu)=R_{A}(\mu)+\lambda \frac{R_{A}(\lambda)-R_{A}(\mu)}{\lambda-\mu}=\frac{\lambda R_{A}(\lambda)-\mu R_{A}(\mu)}{\lambda-\mu}, \quad \lambda, \mu \in \varrho(A) .
$$

It remains to prove (i) and (ii). As to (ii), it suffices to show this for $r=1$. If $\{f, g\} \in R_{A}(\lambda) A$, then there is an $h \in \mathbf{K}$ such that $\{f, h\} \in A$ and $\{h, g\} \in R_{A}(\lambda)$. Hence $g=R_{A}(\lambda) h=\left(I+\lambda R_{A}(\lambda)\right) f$ and $\{f, g\} \in I+\lambda R_{A}(\lambda)$. Therefore $R_{A}(\lambda) A \subset$ $I+\lambda R_{A}(\lambda)$. The second inclusion in (ii) with $r=1$ is clear for $\lambda=0$. If $\lambda \neq 0$ and $\{f, g\} \in I+\lambda R_{A}(\lambda)$ then $\{\lambda f, g-f\} \in R_{A}(\lambda)$ and $\{g-f, \lambda g\} \in A$, so that $\{\lambda f, \lambda g\} \in A R_{A}(\lambda)$. Thus we have $I+\lambda R_{A}(\lambda) \subset A R_{A}(\lambda)$. First we prove (i) in the case $r=1$. If $\{f, g\} \in A$, then $R_{A}(\lambda)(g-\lambda f)=f$. If $\varphi=g-\lambda f$, then $f=R_{A}(\lambda) \varphi$ and $\left(I+\lambda R_{A}(\lambda)\right) \varphi=$ $\left(I+\lambda R_{A}(\lambda)\right)(g-\lambda f)=g$. Hence $A \subset\left\{\left\{R_{A}(\lambda) \varphi,\left(I+\lambda R_{A}(\lambda)\right) \varphi\right\} \mid \varphi \in \mathbf{K}\right\}, \lambda \in \varrho(A)$. The reverse inclusion is obvious. Now suppose (i) holds for $r=1, \ldots, p-1$. Suppose $\{f, g\} \in A^{p}$, then for some $h_{1}, \ldots, h_{p-1} \in \mathbf{K}$, we have $\left\{f, h_{1}\right\} \in A,\left\{h_{1}, h_{2}\right\} \in A, \ldots$, 
$\left\{h_{p-2}, h_{p-1}\right\} \in A,\left\{h_{p-1}, g\right\} \in A$. This implies for $\varphi_{1}, \ldots, \varphi_{p} \in \mathbf{K}$ that

$$
\begin{array}{ll}
f=R_{A}(\lambda) \varphi_{1}, & h_{1}=\left(I+\lambda R_{A}(\lambda)\right) \varphi_{1}, \\
h_{1}=R_{A}(\lambda) \varphi_{2}, \quad h_{2}=\left(I+\lambda R_{A}(\lambda)\right) \varphi_{2}, \\
\ldots \ldots \ldots \ldots \ldots \ldots \ldots \ldots \ldots \ldots \ldots \ldots \ldots \ldots \ldots \ldots \ldots \ldots \ldots \ldots \ldots \ldots \ldots \ldots \ldots \ldots \ldots \ldots \ldots \ldots \ldots \ldots \\
h_{p-2}=R_{A}(\lambda) \varphi_{p-1}, & h_{p-1}=\left(I+\lambda R_{A}(\lambda)\right) \varphi_{p-1}, \\
h_{p-1}=R_{A}(\lambda) \varphi_{p}, \quad g=\left(I+\lambda R_{A}(\lambda)\right) \varphi_{p} .
\end{array}
$$

This shows $\left\{\varphi_{i}, \varphi_{i+1}\right\} \in A$ for $i=1, \ldots, p-1$, and thus $\left\{\varphi_{1}, \varphi_{p}\right\} \in A^{p-1}$ so that for some $\chi \in \mathbf{K} \quad \varphi_{1}=R_{A}(\lambda)^{p-1} \chi, \quad \varphi_{p}=\left(I+\lambda R_{A}(\lambda)\right)^{p-1} \chi$. We conclude $f=R_{A}(\lambda)^{p} \chi$, $g=\left(I+\lambda R_{A}(\lambda)\right)^{p} \chi$ and thus

$$
A^{p} \subset\left\{\left\{R_{A}(\lambda)^{p} \chi,\left(I+\lambda R_{A}(\lambda)\right)^{p} \chi\right\} \mid \chi \in \mathbf{K}\right\}, \quad \lambda \in \varrho(A) .
$$

On the other hand we have for all $\chi \in \mathbf{K}$

while

$$
\left\{R_{A}(\lambda)^{p-1} R_{A}(\lambda) \chi,\left(I+\lambda R_{A}(\lambda)\right)^{p-1} R_{A}(\lambda) \chi\right\} \in A^{p-1},
$$

so that

which shows

$$
\left\{R_{A}(\lambda)\left(I+\lambda R_{A}(\lambda)\right)^{p-1} \chi,\left(I+\lambda R_{A}(\lambda)\right)^{p} \chi\right\} \in A,
$$

$$
\left\{R_{A}(\lambda)^{p} \chi,\left(I+\lambda R_{A}(\lambda)\right)^{p} \chi\right\} \in A^{p}
$$

$$
\left\{\left\{R_{A}(\lambda)^{p} \chi,\left(I+\lambda R_{A}(\lambda)\right)^{p} \chi\right\} \mid \chi \in \mathbf{K}\right\} \subset A^{p}, \quad \lambda \in \varrho(A) .
$$

This completes the proof.

Next we show for any subspace $A \subset \mathbf{K}^{2}$ and $r \in \mathbf{N}$ we have

$$
\{f, g\} \in(A-\lambda)^{r} \Leftrightarrow f=R_{A}(\lambda)^{r} g, \quad \lambda \in \varrho(A) .
$$

To see this let $\{f, g\} \in(A-\lambda)^{r}$, then for $g_{1}, \ldots, g_{r-1} \in \mathbf{K}$ we have $\left\{f, g_{1}\right\} \in A-\lambda$, $\left\{g_{1}, g_{2}\right\} \in A-\lambda, \ldots,\left\{g_{r-2}, g_{r-1}\right\} \in A-\lambda, \quad\left\{g_{r-1}, g\right\} \in A-\lambda, \quad$ so that $g_{r-1}=R_{A}(\lambda) g$, $g_{r-2}=R_{A}(\lambda) g_{r-1}, \ldots, g_{1}=R_{A}(\lambda) g_{2}, f=R_{A}(\lambda) g_{1}$, which implies $f=R_{A}(\lambda)^{r} g$. The proof is complete by reversing the above argument.

If $\mathbf{K}$ is a linear space, $A$ a linear relation in $\mathbf{K}^{2}$ and if $p$ is a polynomial, $p(\lambda)=\sum_{k=0}^{n} b_{k} \lambda^{k}, b_{k} \in \mathbf{C}, b_{n} \neq 0$, we may define the linear relation $p(A)$ in the obvious way

$$
p(A)=\left\{\{f, g\} \in \mathbf{K}^{2} \mid\left\{f, g_{i}\right\} \in b_{i} A^{i}, i=0, \ldots, n, \sum_{i=0}^{n} g_{i}=g\right\} .
$$

With $\alpha \in \mathbf{C}$, we can write $p$ in a different way: $p(\lambda)=\sum_{k=0}^{n} c_{k}(\lambda-\alpha)^{k}, c_{n}=b_{n} \neq 0$, and it has been proven (see [1]), that

$$
p(A)=\sum_{k=0}^{n} c_{k}(A-\alpha)^{k} .
$$

We now return to the Banach space situation. 
Proposition 2.3. Let $A \subset \mathbf{K}^{2}$ be a subspace with $\varrho(A) \neq \emptyset$ and let $p$ be $a$ polynomial of degree $n$

Then

$$
p(\lambda)=\sum_{k=0}^{n} c_{k}(\lambda-\alpha)^{k}, \quad c_{n} \neq 0, \quad \lambda \in \varrho(A) .
$$

$$
\{f, g\} \in p(A) \Leftrightarrow \sum_{k=0}^{n} c_{k} R_{A}(\alpha)^{n-k} f=R_{A}(\alpha)^{n} g, \quad \alpha \in \varrho(A) .
$$

Proof. In order to show this we let $\{f, g\} \in p(A)$, then for $g_{0}, \ldots, g_{n} \in \mathbf{K}$ we have $\left\{f, g_{k}\right\} \in c_{k}(A-\alpha)^{k}$, or $\left\{c_{k} f, g_{k}\right\} \in(A-\alpha)^{k}$ and $\sum_{k=0}^{n} g_{k}=g$. By (2.3) this implies $c_{k} f=R_{A}(\alpha)^{k} g_{k}$ and $c_{k} R_{A}(\alpha)^{n-k} f=R_{A}(\alpha)^{n} g_{k}$ so that

$$
\sum_{k=0}^{n} c_{k} R_{A}(\alpha)^{n-k} f=R_{A}(\alpha)^{n} g .
$$

To prove the converse we define $\varphi_{0}, \ldots, \varphi_{n} \in \mathbf{K}$ and $\psi_{0}, \ldots, \psi_{n} \in \mathbf{K}$ by

and

$$
\begin{gathered}
\varphi_{0}=f, \\
\varphi_{j}=\frac{1}{c_{n}}\left(R_{A}(\alpha)^{n-j} g-\sum_{k=0}^{n-1} c_{k} R_{A}(\alpha)^{n-k-1} \varphi_{j-1}\right), \quad j=1, \ldots, n,
\end{gathered}
$$

$$
\psi_{j}=\sum_{k=0}^{n} c_{k} R_{A}(\alpha)^{n-k} \varphi_{j}-R_{A}(\alpha)^{n-j} g, \quad j=0, \ldots, n .
$$

Then we have

We claim

$$
c_{n}\left(\varphi_{j-1}-R_{A}(\alpha) \varphi_{j}\right)=\psi_{j-1}, \quad j=1, \ldots, n
$$

$$
\psi_{j}=0, \quad j=0, \ldots, n-1 \text {. }
$$

Clearly this is true for $j=0$. Assume it is true for $j=p-1,1 \leqq p \leqq n-1$. Then

$$
\begin{aligned}
\psi_{p} & =c_{n}\left(\varphi_{p}-R_{A}(\alpha) \varphi_{p+1}\right) \\
& =R_{A}(\alpha)^{n-p} g-\sum_{k=0}^{n-1} c_{k} R_{A}(\alpha)^{n-k-1} \varphi_{p-1}-R_{A}(\alpha)^{n-p} g+\sum_{k=0}^{n-1} c_{k} R_{A}(\alpha)^{n-k} \varphi_{p} \\
& =-\sum_{k=0}^{n-1} c_{k} R_{A}(\alpha)^{n-k-1}\left(\varphi_{p-1}-R_{A}(\alpha) \varphi_{p}\right)=0 .
\end{aligned}
$$

It follows that

This implies that

and

$$
\begin{array}{ll}
R_{A}(\alpha) \varphi_{j}=\varphi_{j-1}, & j=1, \ldots, n . \\
R_{A}(\alpha)^{n-j} \varphi_{n}=\varphi_{j}, & j=0, \ldots, n, \\
R_{A}(\alpha)^{j} \varphi_{j}=f, & j=0, \ldots, n,
\end{array}
$$

and also that

$$
\begin{aligned}
R_{A}(\alpha) \psi_{n} & =\sum_{k=0}^{n} c_{k} R_{A}(\alpha)^{n-k+1} \varphi_{n}-R_{A}(\alpha) g \\
& =\sum_{k=0}^{n} c_{k} R_{A}(\alpha)^{n-k} \varphi_{n-1}-R_{A}(\alpha) g \\
& =\psi_{n-1}=0 .
\end{aligned}
$$


Hence we obtain

and

$$
\left\{f, \varphi_{j}\right\} \in(A-\alpha)^{j}, \quad j=0, \ldots, n,
$$

$$
\sum_{k=0}^{n} c_{k} \varphi_{k}=g+\psi_{n} .
$$

Therefore $\left\{f, g+\psi_{n}\right\} \in p(A)$ and as $\psi_{n} \in v\left(R_{A}(\alpha)\right)=A(0)$ implies that $\left\{0, \psi_{n}\right\} \in p(A)$ we obtain $\{f, g\} \in p(A)$. This completes the proof.

Proposition 2.4. Let $A \subset \mathbf{K}^{2}$ be a subspace with $\varrho(A) \neq \emptyset$, then

(i) $p(A)$ is a closed linear relation,

(ii) $R_{A}(\lambda)^{n} p(A) R_{A}(\mu)^{n} \in[\mathrm{K}], \lambda, \mu \in \varrho(A)$.

Proof. (i) is an immediate consequence of Proposition 2.3. As to (ii) we observe that

$$
\begin{aligned}
& R_{A}(\lambda)^{n} p(A) R_{A}(\mu)^{n}=\sum_{k=0}^{n} b_{k} R_{A}(\lambda)^{n} A^{k} R_{A}(\mu)^{n} \\
& =\sum_{k=0}^{n} b_{k} R_{A}(\lambda)^{n-k} R_{A}(\lambda)^{k} A^{k} R_{A}(\mu)^{k} R_{A}(\mu)^{n-k} \\
& =\sum_{k=0}^{n} b_{k} R_{A}(\lambda)^{n-k}\left(I+\lambda R_{A}(\lambda)\right)^{k} R_{A}(\mu)^{k} R_{A}(\mu)^{n-k} \\
& =\sum_{k=0}^{n} b_{k} R_{A}(\lambda)^{n-k}\left(I+\lambda R_{A}(\lambda)\right)^{k} R_{A}(\mu)^{n},
\end{aligned}
$$

where we have used Theorem 2.2.

If $A$ is a closed operator in $\mathbf{K}$, with $\varrho(A) \neq \emptyset$, then a proof of (i) can be found for instance in [7, p. 602]. Our proof of the general case is modelled on that argument, and is different from the reasoning in [1].

Let $A \subset \mathbf{K}^{2}$ be a linear relation. We recall the definitions of the continuous spectrum $\sigma_{c}(A)$ and the residual spectrum $\sigma_{r}(A)$ :

$$
\begin{aligned}
& \sigma_{c}(A)=\left\{\lambda \in \mathbf{C} \mid \mathbf{R}(A-\lambda)^{c}=\mathbf{K}, \mathbf{R}(A-\lambda) \neq \mathbf{K}, v(A-\lambda)=\{0\}\right\}, \\
& \sigma_{r}(A)=\left\{\lambda \in \mathbf{C} \mid \mathbf{R}(A-\lambda)^{c} \neq \mathbf{K}, v(A-\lambda)=\{0\}\right\} .
\end{aligned}
$$

Sometimes it is useful to include $\infty$ in the resolvent set or in the spectrum. Therefore we define, cf. [5],

$$
\begin{array}{llllll}
\varrho(A)=\varrho(A) \cup\{\infty\} & \text { if } & 0 \in \varrho\left(A^{-1}\right), & =\varrho(A) & \text { if } & 0 \notin \varrho\left(A^{-1}\right), \\
\tilde{\sigma}(A)=\sigma(A) \cup\{\infty\} & \text { if } & 0 \in \sigma\left(A^{-1}\right), & =\sigma(A) & \text { if } & 0 \notin \sigma\left(A^{-1}\right), \\
\tilde{\sigma}_{p}(A)=\sigma_{p}(A) \cup\{\infty\} & \text { if } & 0 \in \sigma_{p}\left(A^{-1}\right), & =\sigma_{p}(A) & \text { if } & 0 \notin \sigma_{p}\left(A^{-1}\right), \\
\tilde{\sigma}_{c}(A)=\sigma_{c}(A) \cup\{\infty\} & \text { if } & 0 \in \sigma_{c}\left(A^{-1}\right), & =\sigma_{c}(A) & \text { if } & 0 \notin \sigma_{c}\left(A^{-1}\right), \\
\tilde{\sigma}_{r}(A)=\sigma_{r}(A) \cup\{\infty\} & \text { if } & 0 \in \sigma_{r}\left(A^{-1}\right), & =\sigma_{r}(A) & \text { if } & 0 \notin \sigma_{r}\left(A^{-1}\right) .
\end{array}
$$

In particular note that for a subspace $A \subset \mathbf{K}^{2}$ we have

$$
\infty \in \tilde{\varrho}(A) \Leftrightarrow A \in[\mathbf{K}] .
$$


Now let $M$ be an invertible $2 \times 2$ matrix with complex entries. It is easy to see that for any linear relation $A \subset \mathbf{K}^{2}$ we have

$A$ is closed $\Leftrightarrow M A$ is closed.

Note that for a subspace $A \subset \mathbf{K}^{2}$ we have

$$
\mu \in \varrho(A) \Leftrightarrow C_{\mu}(A) \in[\mathbf{K}], \quad \mu \in \mathbf{C} \backslash \mathbf{R},
$$

and for a subspace $U \subset \mathbf{K}^{2}$ we have

$$
\mu \in \varrho\left(F_{\mu}(U)\right) \Leftrightarrow U \in[\mathbf{K}], \quad \mu \in \mathbf{C} \backslash \mathbf{R} .
$$

For $\mu \in \mathbf{C}$ we introduce a homeomorphism $\Theta$ from the extended complex plane into itself with the usual topology by

$$
\Theta(\lambda)=\frac{\lambda-\bar{\mu}}{\lambda-\mu}, \quad \lambda \neq \mu, \quad \Theta(\infty)=1, \quad \Theta(\mu)=\infty .
$$

Proposition 2.5. Let $A \subset \mathbf{K}^{2}$ be a linear relation, and let $\mu \in \varrho(A) \backslash \mathbf{R}$. Then $\Theta$ gives a one-to-one correspondence between

(i) $\tilde{\varrho}(A)$ and $\tilde{\varrho}\left(C_{\mu}(A)\right)$,

(ii) $\tilde{\sigma}(A)$ and $\sigma\left(C_{\mu}(A)\right)$,

(iii) $\tilde{\sigma}_{p}(A)$ and $\sigma_{p}\left(C_{\mu}(A)\right)$,

(iv) $\tilde{\sigma}_{c}(A)$ and $\sigma_{c}\left(C_{\mu}(A)\right)$,

(v) $\tilde{\sigma}_{r}(A)$ and $\sigma_{r}\left(C_{\mu}(A)\right)$.

Proof. This is based on the identity

$$
\left(C_{\mu}(A)-\frac{\lambda-\bar{\mu}}{\lambda-\mu}\right)^{-1}=\frac{\mu-\lambda}{\mu-\bar{\mu}}\left(I+(\lambda-\mu)(A-\lambda)^{-1}\right), \quad \mu \in \mathbf{C} \backslash \mathbf{R}, \lambda \neq \mu .
$$

For the sake of completeness we include a couple of results, due to Arens [1].

Proposition 2.6. Let $A, B$ be subspaces, such that $A B=B A$. Then

$$
(A B)^{-1} \in[\mathbf{K}] \Leftrightarrow A^{-1} \in[\mathbf{K}] \text { and } B^{-1} \in[\mathbf{K}] \text {. }
$$

Proof. If $A^{-1}, B^{-1} \in[\mathbf{K}]$, then $(A B)^{-1}=B^{-1} A^{-1} \in[\mathbf{K}]$. Conversely $(A B)^{-1} \in[\mathbf{K}]$ and $A B=B A$ imply $\mathbf{R}(A B)=\mathbf{R}(B A)=\mathbf{K}$ and $v(A B)=v(B A)=\{0\}$. Hence we find $\mathbf{R}(A)=\mathbf{K}, v(A)=\{0\}$ or equivalently $A^{-1} \in[\mathbf{K}]$, and also $B^{-1} \in[\mathbf{K}]$.

Now let $p$ be a complex-valued polynomial of degree $n$, and let $\mu \in \mathbf{C}$. Then

$$
p(\lambda)-p(\mu)=c\left(\lambda-\mu_{1}\right) \ldots\left(\lambda-\mu_{n}\right),
$$

where $p\left(\mu_{i}\right)=p(\mu), i=1, \ldots, n$. Hence if $A \subset \mathbf{K}^{2}$ is a subspace, then

This implies

$$
p(A)-p(\mu)=c\left(A-\mu_{1}\right) \ldots\left(A-\mu_{n}\right) .
$$

$$
p(\mu) \in \sigma(p(A)) \Leftrightarrow \mu_{i} \in \sigma(A) \text { for some } i=1, \ldots, n .
$$

Proposition 2.7. If $A \subset \mathbf{K}^{2}$ is a subspace and $p$ a polynomial, then

$$
\sigma(p(A))=p(\sigma(A)) \text {. }
$$




\section{An operational calculus for subspaces}

We present an operational calculus for subspaces along the lines of [14] or [7, p. 601], by reducing the problem to one for the resolvent operator. If $A \in[\mathbf{K}]$ we define $\mathbf{F}(A)$ to be the set of all complex-valued functions, which are analytic in some neighbourhood of the compact set $\sigma(A)$. If $A \subset \mathbf{K}^{2}$ is a subspace such that $A \notin[\mathbf{K}]$, then $\mathbf{F}(A)$ denotes the set of all complex-valued functions, which are analytic in some neighbourhood of $\tilde{\sigma}(A)$.

For a given $\alpha \in C$ we set up a homeomorphism $\Phi$ from the extended complex plane into itself with the usual topology by

$$
\Phi(\lambda)=\frac{1}{\lambda-\alpha}, \quad \lambda \neq \alpha, \quad \Phi(\alpha)=\infty, \quad \Phi(\infty)=0 .
$$

Proposition 3.1. Let $A \subset \mathbf{K}^{2}$ be a subspace with $\varrho(A) \neq \emptyset$. If $\alpha \in \varrho(A)$, then $\Phi$ gives a one-to-one correspondence between
(i) $\tilde{\varrho}(A)$ and $\tilde{\varrho}\left((A-\alpha)^{-1}\right)$,
(ii) $\tilde{\sigma}(A)$ and $\sigma\left((A-\alpha)^{-1}\right)$,
(iii) $\tilde{\sigma}_{p}(A)$ and $\sigma_{p}\left((A-\alpha)^{-1}\right)$,
(iv) $\tilde{\sigma}_{c}(A)$ and $\sigma_{c}\left((A-\alpha)^{-1}\right)$,
(v) $\tilde{\sigma}_{r}(A)$ and $\sigma_{r}\left((A-\alpha)^{-1}\right)$,
(vi) $\mathbf{F}(A)$ and $\mathbf{F}\left((A-\alpha)^{-1}\right)$, in the sense that

$$
f=\varphi \circ \Phi^{-1}, \quad f \in \mathbf{F}(A), \quad \varphi \in \mathbf{F}((A-\alpha))^{-1} .
$$

Proof. All items except (vi) follow from the identity

$$
(A-\lambda)^{-1}=-\frac{1}{\lambda-\alpha} I-\frac{1}{(\lambda-\alpha)^{2}}\left((A-\alpha)^{-1}-\frac{1}{\lambda-\alpha}\right)^{-1}, \quad \lambda \neq \alpha,
$$

which we have given in Section 2. Item (vi) is straightforward.

If $A \in[\mathbf{K}]$ and $f \in \mathbf{F}(A)$, then we define $f(A)$ by

$$
f(A)=-\frac{1}{2 \pi i} \int_{\Gamma} f(\lambda) R_{A}(\lambda) d \lambda,
$$

where $\Gamma$ is a suitable contour, surrounding the spectrum $\sigma(A)$, cf. [7, p. 601] and [14]. If $A \subset \mathbf{K}^{2}$ is a subspace with $\varrho(A) \neq \emptyset$ and $A \notin[\mathbf{K}]$, then we define for $f \in \mathbf{F}(A)$,

$$
f(A)=f(\infty) I-\frac{1}{2 \pi i} \int_{\Gamma} f(\lambda) R_{A}(\lambda) d \lambda,
$$

where again $\Gamma$ is a suitable contour, surrounding the extended spectrum $\tilde{\sigma}(A)$. This definition is the same as in [7, p. 601] when $A$ is a closed operator. It follows that $f(A) \in[\mathbf{K}]$ and we have the usual properties. The mapping $f \rightarrow f(A)$ is an algebra 
homomorphism from (the algebra of equivalence classes of) $\mathbf{F}(A)$ into [K], which preserves the unit element. We collect some useful properties in the following proposition.

Proposition 3.2. Let $A \subset \mathbf{K}^{2}$ be a subspace with $\varrho(A) \neq \emptyset$. Then

(i) $\sigma(f(A))=f(\tilde{\sigma}(A))$.

(ii) If $f \in \mathbf{F}(A), g \in \mathbf{F}(f(A))$ and $F=g \circ f$, then

$$
F \in \mathbf{F}(A) \text { and } F(A)=g(f(A)) \text {. }
$$

Let $f, g \in \mathbf{F}(A)$, such that for some $\alpha \in \varrho(A) \quad g(\lambda)=(\lambda-\alpha)^{n} f(\lambda)$. Then it follows that $R_{A}(\alpha)^{n} g(A)=f(A)$, which implies that $f(A)$ maps $\mathbf{K}$ into $\mathbf{D}\left(A^{n}\right)$.

If $\sigma \subset \sigma(A)$ is a bounded spectral set of $A$, and

$$
E_{A}(\sigma)=-\frac{1}{2 \pi i} \int_{\Gamma} R_{A}(\lambda) d \lambda,
$$

where $\Gamma$ is a closed contour in $\varrho(A)$ such that $\sigma$ is inside $\Gamma$ and $\sigma(A) \backslash \sigma$ is outside $\Gamma$, then the above remarks show that $E_{A}(\sigma)$ is a projection, the so-called Rieszprojector. It follows that $E_{A}(\sigma)$ maps $\mathbf{K}$ into $\mathbf{D}\left(A^{n}\right), n \in \mathbf{N}$.

Remark. Let $A \subset \mathbf{K}^{2}$ be a subspace with $\varrho(A) \neq \emptyset$, and let $M=\left(\begin{array}{ll}\alpha & \beta \\ \gamma & \delta\end{array}\right)$ be such that $\beta \neq 0$ and such that $-\alpha / \beta \in \varrho(A)$. It is easy to see that

$$
M A=\frac{\delta}{\beta} I-\frac{\operatorname{det} M}{\beta^{2}} R_{A}\left(-\frac{\alpha}{\beta}\right) .
$$

Now suppose $\infty \in \tilde{\sigma}(A)$ or equivalently $A \notin[\mathbf{K}]$, and consider

$$
f(\lambda)=\frac{\delta \lambda+\gamma}{\beta \lambda+\alpha}, \quad \lambda \neq-\frac{\alpha}{\beta} .
$$

Then it follows that $f \in \mathbf{F}(A)$, and

$$
f(A)=M A .
$$

\section{Definitizable subspaces}

In this section we let $\mathbf{K}$ be a Krein space with inner product [, ], see [2]. We recall the definition of the adjoint $A^{+}$for a linear relation $A \subset \mathbf{K}^{2}$ :

$$
A^{+}=\left\{\{f, g\} \in \mathbf{K}^{2} \mid[g, h]=[f, k] \text { for all }\{h, k\} \in A\right\} .
$$

We note that $M A^{+}=(\bar{M} A)^{+}$, for any invertible $2 \times 2$ matrix with complex entries. Here the matrix $\bar{M}$ is defined by

$$
\bar{M}=\left(\begin{array}{ll}
\bar{\alpha} & \bar{\beta} \\
\bar{\gamma} & \bar{\delta}
\end{array}\right) \quad \text { if } \quad M=\left(\begin{array}{ll}
\alpha & \beta \\
\gamma & \delta
\end{array}\right) .
$$


If $A \subset \mathbf{K}^{2}$ is a subspace and $\mu \in \mathbf{C}$ is an isolated point of $\sigma(A)$, then we have a Laurent expansion around $\mu$ :

$$
(A-\lambda)^{-1}=\sum_{k=-\infty}^{\infty}(\mu-\lambda)^{k} A_{k}, \quad A_{k} \in[\mathbf{K}],
$$

and hence around $\bar{\mu}$ we have

$$
\left(A^{+}-\lambda\right)^{-1}=\sum_{k=-\infty}^{\infty}(\bar{\mu}-\lambda)^{k} A_{k}^{+} .
$$

In particular the Jordan structures around the corresponding rootspaces are the same.

More generally, if $\sigma$ is a bounded spectral set of a subspace $A \subset \mathbf{K}^{2}$, then for the corresponding Riesz projector $E_{A}(\sigma)$ we find

$$
E_{A}(\sigma)^{+}=E_{A^{+}}\left(\sigma^{*}\right) \text {. }
$$

Then the subspaces $E_{A}(\sigma) \mathbf{K}$ and $E_{A^{+}}\left(\sigma^{*}\right) \mathbf{K}$ are in duality with respect to the inner product [, ], see [12].

For the sake of completeness we state the following result.

Proposition 4.1. Let $A$ be a selfadjoint subspace in $\mathbf{K}^{2}$ and let $\sigma \subset \sigma(A)$ be a bounded spectral set. Then

(i) $\sigma=\sigma^{*} \quad \Rightarrow \quad E_{A}(\sigma)=E_{A}(\sigma)^{+}$,

(ii) $\sigma \cap \sigma^{*}=\emptyset \Rightarrow E_{A}(\sigma)^{+} E_{A}(\sigma)=0$.

A selfadjoint subspace $A \subset \mathbf{K}^{2}$ is definitizable if $\varrho(A) \neq \emptyset$ and if there exists a real polynomial $p$ such that $p(A) \geqq 0$ in the sense that

$$
[g, f] \geqq 0 \quad \text { for all }\{f, g\} \in p(A) .
$$

This definition was given by Langer in [11] for the case of operators.

Proposition 4.2. Let $A$ be a selfadjoint subspace in $\mathbf{K}^{2}$ with $\varrho(A) \neq 0$, and $\alpha \in \varrho(A)$. Let $p$ be a real polynomial of degree $n$. Then

$$
p(A) \geqq 0 \Leftrightarrow R_{A}(\alpha)^{n} p(A) R_{A}(\bar{\alpha})^{n} \geqq 0 .
$$

Proof. We assume $p(A) \geqq 0$. Let $\{f, g\} \in R_{A}(\alpha)^{n} p(A) R_{A}(\bar{\alpha})^{n}$, then for some $h, k \in \mathbf{K}$, we have

so that

$$
\{f, h\} \in R_{A}(\bar{\alpha})^{n}, \quad\{h, k\} \in p(A), \quad\{k, g\} \in R_{A}(\alpha)^{n},
$$

$$
[g, f]=\left[R_{A}(\alpha)^{n} k, f\right]=\left[k, R_{A}(\bar{\alpha})^{n} f\right]=[k, h] \geqq 0,
$$

which shows one half of the proposition. Now we assume $R_{A}(\alpha)^{n} p(A) R_{A}(\bar{\alpha})^{n} \geqq 0$. Let $\{h, k\} \in p(A)$. Now $\mathbf{D}(p(A)) \subset \mathbf{D}\left(A^{n}\right)$ and an application of Proposition 2.2 shows $h=R_{A}(\bar{\alpha})^{n} \varphi$ for some $\varphi \in \mathbf{K}$. Hence $\{\varphi, h\} \in R_{A}(\bar{\alpha})^{n}, \quad\{h, k\} \in p(A)$ and we define $\psi \in \mathbf{K}$ by $\psi=R_{A}(\alpha)^{n} k$. Then $\{\varphi, \psi\} \in R_{A}(\alpha)^{n} p(A) R_{A}(\bar{\alpha})^{n}$ and

$$
[k, h]=\left[k, R_{A}(\bar{\alpha})^{n} \varphi\right]=\left[R_{A}(\alpha)^{n} k, \varphi\right]=[\psi, \varphi] \geqq 0 .
$$

This completes the proof. 
Corollary. Let $A$ be a selfadjoint subspace in $\mathbf{K}^{2}$ with $\varrho(A) \neq \emptyset$ and $\alpha \in \varrho(A)$. Let $p$ be a real polynomial of degree $n$ and define

$$
q(\lambda)=p(\lambda)(\lambda-\alpha)^{-n}(\lambda-\bar{\alpha})^{-n}, \quad \lambda \neq \alpha, \bar{\alpha} .
$$

Then $q$ is analytic in the extended complex plane with poles in $\alpha, \bar{\alpha}$, and in particular $q \in \mathbf{F}(A)$. We have $A$ definitizable by means of the polynomial $p$ if and only if $q(A) \geqq 0$.

Proof. A simple calculation shows that

$$
q(A)=R_{A}(\alpha)^{n} p(A) R_{A}(\bar{\alpha})^{n} .
$$

Proposition 4.3. If the selfadjoint subspace $A$ in $\mathbf{K}^{2}$ is definitizable, then $A^{-1}$ is also definitizable.

Proof. If $A$ is selfadjoint, then also $A^{-1}$ is selfadjoint. From $\varrho(A) \neq \emptyset$ it follows that $\varrho\left(A^{-1}\right) \neq \emptyset$, since

$$
\left(A-\frac{1}{\mu}\right)^{-1}=-\mu I-\mu^{2}\left(A^{-1}-\mu\right)^{-1}, \quad \mu \in \mathbf{C} \backslash\{0\} .
$$

This also shows that

$$
\alpha \in \varrho(A) \backslash\{0\} \Rightarrow \beta=\alpha^{-1} \in \varrho\left(A^{-1}\right) .
$$

Suppose $A$ is definitizable by means of the polynomial

$$
p(\lambda)=\sum_{j=0}^{n} b_{j} \lambda^{j}, \quad b_{j} \in \mathbf{R}, \quad b_{n} \neq 0 .
$$

Then we define the polynomial

$$
\tilde{p}(\lambda)=\sum_{j=0}^{n} b_{j} \lambda^{2 n-j},
$$

and we observe that with

$$
q(\lambda)=p(\lambda)(\lambda-\alpha)^{-n}(\lambda-\bar{\alpha})^{-n}, \quad \lambda \neq \alpha, \bar{\alpha}, \quad \alpha \in \varrho(A) \backslash\{0\},
$$

we have with $\beta=\alpha^{-1}$,

$$
q\left(\frac{1}{\lambda}\right)=|\alpha|^{2 n} \tilde{p}(\lambda)(\lambda-\beta)^{-n}(\lambda-\bar{\beta})^{-n} .
$$

This shows that $A^{-1}$ is definitizable by means of the polynomial $\tilde{p}$ if and only if $\tilde{q}\left(A^{-1}\right) \geqq 0$, where $\tilde{q}(\lambda)=q(1 / \lambda)$. However this follows from $\tilde{q}\left(A^{-1}\right)=q(A)$, using the relation

$$
R_{A^{-1}}(\lambda)=-\frac{1}{\lambda} I-\frac{1}{\lambda^{2}} R_{A}\left(\frac{1}{\lambda}\right), \quad \lambda \in \varrho\left(A^{-1}\right),
$$

and the operational calculus.

Proposition 4.4. Let $\mathbf{K}$ be a Pontryagin space and let $A$ be a selfadjoint subspace in $\mathbf{K}^{2}$ with $\varrho(A) \neq \emptyset$. Then $A$ is definitizable. 
Proof. Choose $\alpha \in \varrho(A) \backslash \mathbf{R}$, then $C_{\alpha}(A)$ is a unitary operator in [K]. Remember that with

we have

$$
\theta(\lambda)=\frac{\lambda-\bar{\alpha}}{\lambda-\alpha}, \quad \lambda \neq \alpha,
$$

$$
C_{\alpha}(A)=\theta(A),
$$

see the operational calculus in Section 3. Since $\mathbf{K}$ is a Pontryagin space there is a function $g \in \mathbf{F}\left(C_{\alpha}(A)\right)$ such that

$$
g\left(C_{\alpha}(A)\right) \geqq 0,
$$

see [11, p. 52], and with the additional property, see [11, p. 57],

$$
(g \circ \theta)(\lambda)=g(\theta(\lambda))=p(\lambda)(\lambda-\alpha)^{-n}(\lambda-\bar{\alpha})^{-n}, \quad \lambda \neq \alpha, \bar{\alpha},
$$

where $p$ is a real polynomial of degree $n$. Using the operational calculus it can be seen that

$$
(g \circ \theta)(A)=g(\theta(A))=g\left(C_{\alpha}(A)\right) \geqq 0 .
$$

The Corollary to Proposition 4.2 now gives the desired result.

We close this section with a result about non-negative relations. A linear relation $A \subset \mathbf{K}^{2}$ is non-negative, $A \geqq 0$, if

$$
[g, f] \geqq 0, \quad\{f, g\} \in A .
$$

Such a linear relation is necessarily symmetric. If $A=A^{+} \subset \mathbf{K}^{2}$ with $\varrho(A) \neq \emptyset$, we have

$$
A \geqq 0 \Leftrightarrow \frac{\alpha R_{A}(\alpha)-\bar{\alpha} R_{A}(\bar{\alpha})}{\alpha-\bar{\alpha}} \geqq 0, \quad \alpha \in \varrho(A) \backslash \mathbf{R} .
$$

Proposition 4.5. Let $A$ be a selfadjoint subspace in $\mathbf{K}^{2}$ and $A \geqq 0$. If $A^{-1} \in[\mathbf{K}]$, then $A$ is a definitizable and

$$
\sigma(A) \subset \mathbf{R} .
$$

Proof. Since $A^{-1} \in[\mathbf{K}]$, we see that $0 \in \varrho(A)$, and therefore the selfadjoint subspace $A$ is definitizable. It is clear that $A$ is non-negative if and only if $A^{-1}$ is nonnegative. Hence we have $A^{-1} \in[\mathbf{K}]$ and $A^{-1} \geqq 0$, which implies $\sigma\left(A^{-1}\right) \subset \mathbf{R}$, see [2, p. 147], and therefore $\sigma(A) \subset \mathbf{R}$.

We note that there are examples of selfadjoint subspaces $A$ in $\mathbf{K}^{2}$, such that $A \geqq 0$ and $\sigma_{c}(A)=\mathbf{C}$. 


\section{The resolvent of a definitizable subspace}

In this section we again let $\mathbf{K}$ be a Kreĭn space. Let $A=A^{+} \subset \mathbf{K}^{2}$ with $\varrho(A) \neq \emptyset$ be definitizable by means of the real polynomial $p$ of degree $n: p(A) \geqq 0$, and define

$$
q(\lambda)=p(\lambda)(\lambda-\alpha)^{-n}(\lambda-\bar{\alpha})^{-n}, \quad \alpha \in \varrho(A), \quad \lambda \neq \alpha, \bar{\alpha} .
$$

We have seen $q \in \mathbf{F}(A)$ and $q(A) \geqq 0$. Next we define for $\lambda, \zeta \neq \alpha, \bar{\alpha}$ the function $Q$ by

Then we obtain

$$
\begin{aligned}
Q(\lambda, \zeta) & =\frac{q(\lambda)-q(\zeta)}{\lambda-\zeta}, & & \lambda \neq \zeta, \\
& =q^{\prime}(\lambda), & & \lambda=\zeta .
\end{aligned}
$$

$$
\begin{aligned}
Q(\lambda, \zeta)= & \frac{(\lambda-\alpha)^{-n}(p(\lambda)-p(\zeta))(\zeta-\bar{\alpha})^{-n}}{\lambda-\zeta}+\frac{(\lambda-\alpha)^{-n} p(\lambda)\left((\lambda-\bar{\alpha})^{-n}-(\zeta-\bar{\alpha})^{-n}\right)}{\lambda-\zeta} \\
& +\frac{\left((\lambda-\alpha)^{-n}-(\zeta-\alpha)^{-n}\right) p(\zeta)(\zeta-\bar{\alpha})^{-n}}{\lambda-\zeta} \\
= & \frac{(\lambda-\alpha)^{-n}(p(\lambda)-p(\zeta))(\zeta-\bar{\alpha})^{-n}}{\lambda-\zeta}-(\lambda-\alpha)^{-n} p(\lambda) \sum_{j=0}^{n-1}(\lambda-\bar{\alpha})^{j-n}(\zeta-\bar{\alpha})^{-j-1} \\
& -(\zeta-\bar{\alpha})^{-n} p(\zeta) \sum_{j=0}^{n-1}(\lambda-\alpha)^{j-n}(\zeta-\alpha)^{-j-1} .
\end{aligned}
$$

In the las term of this identity we write $p(\zeta)=\sum_{k=0}^{n} c_{k}(\zeta-\alpha)^{k}$ and observe that

$$
\begin{aligned}
p(\zeta)(\zeta-\alpha)^{-j-1} & =\sum_{k=0}^{n} c_{k}(\zeta-\alpha)^{k-j-1} \\
& =\sum_{k=0}^{n} \sum_{h=0}^{k-j-1} c_{k}\left(\begin{array}{c}
k-j-1 \\
h
\end{array}\right)(\zeta-\bar{\alpha})^{h}(\bar{\alpha}-\alpha)^{k-j-1-h} .
\end{aligned}
$$

This shows that for each $\lambda$ in the extended plane, with the exception of the points $\alpha, \bar{\alpha}$, the operator $Q(\lambda, A) \in[\mathbf{K}]$ is well-defined and that the mapping $\lambda \rightarrow Q(\lambda, A)$ is holomorphic there. If $\lambda \in \varrho(A), \lambda \neq \alpha, \bar{\alpha}$, then we have

$$
Q(\lambda, A)=(q(A)-q(\lambda)) R_{A}(\lambda) .
$$

Now we proceed as in [12], where densely defined operators $A=A^{+}$are treated. We have

$$
R_{A}(\lambda)=q(\lambda)^{-1} q(A) R_{A}(\lambda)-q(\lambda)^{-1} Q(\lambda, A), \quad \lambda \in \varrho(A), \quad q(\lambda) ; 00 .
$$

Since $q(A) R_{A}(\lambda)=R_{A}(\lambda) q(A), \lambda \in \varrho(A)$, we obtain for each $f \in \mathbf{K}$

$$
\operatorname{Im}\left[q(A) R_{A}(\lambda) f, f\right]=(\operatorname{Im} \lambda)\left[q(A) R_{A}(\lambda) f, R_{A}(\lambda) f\right], \quad \lambda \in \varrho(A),
$$

and hence by Schwarz' inequality

or

$$
|\operatorname{Im} \lambda|\left[q(A) R_{A}(\lambda) f, R_{A}(\lambda) f\right] \leqq\left[q(A) R_{A}(\lambda) f, R_{A}(\lambda) f\right]^{1 / 2}[q(A) f, f]^{1 / 2},
$$

$$
|\operatorname{Im} \lambda|^{2}\left[q(A) R_{A}(\lambda) f, R_{A}(\lambda) f\right] \leqq[q(A) f, f] .
$$


Therefore we obtain for $\lambda \in \mathbf{C}^{+} \cap \varrho(A)$

$$
0 \leqq \operatorname{Im}\left[q(A) R_{A}(\lambda) f, f\right] \leqq \frac{1}{\operatorname{Im} \lambda}[q(A) f, f], \quad f \in \mathbf{K},
$$

which shows that $\lambda \rightarrow\left[q(A) R_{A}(\lambda) f, f\right]$ maps $\mathbf{C}^{+} \cap \varrho(A)$ into $\mathbf{C}^{+}$. This implies that the function $\lambda \rightarrow q(A) R_{A}(\lambda)$ has an analytic extension from all of $\mathbf{C}^{+}$into $\mathbf{C}^{+}$, see for instance [10].

Using a similar observation (given above) for the function $\lambda \rightarrow Q(\lambda, A)$, we see that the mapping $\lambda \rightarrow R_{A}(\lambda)$ has an analytic continuation into

$$
\mathbf{C}^{+} \backslash\left\{\lambda \in \mathbf{C}^{+} \mid p(\lambda)=0\right\},
$$

and hence this set belongs to $\varrho(A)$, cf. [6]. Next we observe for all $\chi, h \in \mathbf{K}$

$$
\begin{aligned}
\left|i \eta\left[q(A) R_{A}(i \eta) \chi, h\right]\right|^{2} & \leqq \eta^{2}\left[q(A) R_{A}(i \eta) \chi, R_{A}(i \eta) \chi\right][q(A) h, h] \\
& \leqq[q(A) \chi, \chi][q(A) h, h],
\end{aligned}
$$

if $\eta \in \mathbf{R}$ is large enough. Now we proceed using standard arguments. If we choose on $\mathbf{K}$ a fundamental decomposition $J$, and define the inner product $(.,)=.[J .,$.$] ,$ then $(\mathbf{K},()$,$) is a Hilbert space and it follows that there exists a non-decreasing left-$ continuous function $F$ on $\mathbf{R}$, whose values are bounded selfadjoint operators in the Hilbert space $(\mathbf{K},()$,$) , such that$

$$
q(A) R_{A}(\lambda)=J \int_{\mathbf{R}} \frac{1}{t-\lambda} d F(t), \quad \lambda \in \varrho(A) .
$$

Proposition 5.1. Under the conditions stated above, we have

$$
R_{A}(\lambda)=\frac{1}{q(\lambda)} J \int_{\mathrm{R}} \frac{1}{t-\lambda} d F(t)-\frac{1}{q(\lambda)} Q(\lambda, A), \quad \lambda \in \varrho(A),
$$

where the integral exists in the strong operator topology. In particular, if $\lambda \in \sigma(A)$ then either $p(\lambda)=0$ or $\lambda \in \mathbf{R}$ is a point of increase of $F$. Also we have in the sense of the Hilbert space $(\mathbf{K},()$,$) that$

$$
F(\infty) \leqq J q(A) .
$$

Note that if $f \in \mathbf{D}(A)$, then $\{f, g\} \in A$ for some $g \in \mathbf{K}$ and

$$
\left(I+\lambda R_{A}(\lambda)\right) f=R_{A}(\lambda) g, \quad \lambda \in \varrho(A) .
$$

Hence for all $h \in \mathbf{K}$, we obtain

which implies

$$
\begin{gathered}
{[q(A) f, h]+i \eta\left[q(A) R_{A}(i \eta) f, h\right]=\left[q(A)\left(I+i \eta R_{A}(i \eta)\right) f, h\right]} \\
=\left[\left(I+i \eta R_{A}(i \eta)\right) f, q(A) h\right]=\left[R_{A}(i \eta) g, q(A) h\right],
\end{gathered}
$$

$$
\begin{aligned}
& \left|[q(A) f, h]+i \eta\left[q(A) R_{A}(i \eta) f, h\right]\right|^{2} \\
\leqq & {[q(A) g, g]\left[q(A) R_{A}(i \eta) h, R_{A}(i \eta) h\right] . }
\end{aligned}
$$


Therefore we conclude

$$
\lim _{\eta \rightarrow \infty}-i \eta\left[q(A) R_{A}(i \eta) f, h\right]=[q(A) f, h], \quad f \in \mathbf{D}(A), \quad h \in \mathbf{K} .
$$

By a limiting procedure we obtain

$$
\lim _{\eta \rightarrow \infty}-i \eta\left[q(A) R_{A}(i \eta) f, h\right]=[q(A) f, h], \quad f \in(\mathbf{D}(A))^{c}, \quad h \in \mathbf{K} .
$$

It is clear that for all $\eta>0$

$$
-i \eta\left[q(A) R_{A}(i \eta) f, h\right]=0, \quad f \in \mathbf{D}(A)^{[\perp]}, \quad h \in \mathbf{K} .
$$

Remark. Let $A=A^{+}$be a definitizable subspace in $\mathbf{K}^{2}$, as above. If $\mathbf{D}(A)^{c}$ is a Krein space and $E: \mathbf{K} \rightarrow \mathbf{D}(A)^{c}$ is the corresponding projection, then $F(\infty)=$ $J q(A) E$.

Some more consequences of the above arguments are formulated in the following proposition. For $\lambda \in \mathbf{C}$ we denote by $k(\lambda)$ its multiplicity as a zero of the fixed definitizing polynomial $p$.

Proposition 5.2. Let $A=A^{+}$be a definitizable subspace in $\mathbf{K}^{2}$, with real definitizing polynomial $p$. Then the non-real spectrum of $A$ consists of a finite number of pairs $\lambda, \bar{\lambda}$. Each isolated spectral point of $A$ is an eigenvalue of finite Riesz index $v(\lambda)=v(\bar{\lambda})$ :

Near the real axis we have

$$
v(\lambda) \leqq \begin{cases}k(\lambda), & \lambda \in \mathbf{C} \backslash \mathbf{R}, \\ k(\lambda)+1, & \lambda \in \mathbf{R} .\end{cases}
$$

$$
\left\|R_{A}(\xi+i \eta)\right\|=o\left(\eta^{-k(\zeta)-1}\right), \quad \xi, \eta \in \mathbf{R}, \eta \rightarrow 0 .
$$

In particular, if $\xi \in \mathbf{R}$ is an eigenvalue of $A$ each corresponding Jordan chain is of length at most $k(\xi)+1$.

\section{References}

[1] ARENS, R.: Operational calculus of linear relations. - Pacific J. Math. 11, 1961, 9-23.

[2] BoGNÁR, J.: Indefinite inner product spaces. - Springer-Verlag, Berlin-Heidelberg-New York, 1974.

[3] Bognár, J.: A proof of the spectral theorem for J-positive operators. - Acta Sci. Math. 45, 1983, 75-80.

[4] BoGNÁR, J.: An approach to the spectral decomposition of $J$-positizable operators. - Preprint.

[5] Coddington, E. A., and H. S. V. DE SNOo: Regular boundary value problems associated with pairs of ordinary differential expressions. - Lecture Notes in Mathematics 858. Springer-Verlag, Berlin-Heidelberg-New York, 1981.

[6] Dijksma, A., and H. S. V. DE SNOo: Symmetric and selfadjoint relations in Kreĭn spaces I. - Operators in indefinite metric spaces, scattering theory and other topics (Bucharest, 1985). Operator Theory: Advances and Applications 24. Birkhäuser Verlag, Basel-Boston, 1987, 144-166. 
[7] Dunford, N., and J. T. Schwartz: Linear operators, Part I. - Interscience Publishers Inc., New York, 1958.

[8] JoNAS, P.: On the functional calculus and the spectral function for definitizable operators in Kreĭn space. - Beiträge Anal. 16, 1981, 121-135.

[9] JoNAs, P.: On spectral distributions of definitizable operators in Kreĭn space. - Spectral Theory (Warsaw, 1977). Banach Center Publ. 8, PWN, Warsaw, 1982, 301-311.

[10] KreǏN, M. G., and H. LANGER: Über einige Fortsetzungsprobleme die eng mit der Theorie hermitescher Operatoren im Raume $\Pi_{z}$ zusammenhängen. I. Einige Funktionenklassen und ihre Darstellungen. - Math. Nachr. 77, 1977, 187-236.

[11] LANGER, H.: Spektraltheorie linearer Operatoren in $J$-Räumen und einige Anwendungen auf die Schar $L(\lambda)=\lambda^{2} I+\lambda B+C$. - Habilitationsschrift. Technische Universität Dresden, 1965.

[12] LANGER, H.: Spectral functions of definitizable operators in Kreĭn spaces. - Functional analysis (Dubrovnik 1981). Lecture Notes in Mathematics 948. Springer-Verlag, BerlinHeidelberg-New York, 1982, 1-46.

[13] Shmul'yan, YU. L.: Transformers of linear relations in $J$-spaces. - Funktsional. Anal. i Prilozhen. 14, 1980, 39-44. [Russian. English translation: Functional Anal. Appl. 14, 1980, 110-113.]

[14] TAYLOR, A. E., and D. C. LAY: Introduction to functional analysis. - Second edition. John Wiley \& Sons, New York-Chichester-Brisbane, 1980.

Rijksuniversiteit Groningen

Wiskunde en Informatica

Postbus 800

9700 AV Groningen

Nederland

Received 2 July 1986 